\title{
Traumatic Abdominal Wall Hernias: Classification, Diagnosis and Management
}

\author{
Andrei Mincu, ${ }^{1}$ Florentina Mușat, ${ }^{2}$ Daniel Ion,,, 2 Alexandra Bolocan,, ${ }^{1,2}$ \\ Dan Nicolae Păduraru, ${ }^{1,2}$ Octavian Andronic ${ }^{1,2}$
}

\begin{abstract}
Traumatic abdominal wall hernias (TAWHs) are uncommon health conditions that involve a disruption of the muscular layers after a blunt abdominal injury. A consensus in the management of TAWHs has not been established, owing to the scarce number of reported cases. These hernias can occur under a broad array of clinical presentations, from stable and asymptomatic patients to emergency situations with associated intra-abdominal injuries. The aetiology of TAWHs is diverse and can occur at all ages, however it is more likely to be reported in children and elderly patients. Clinical findings must be correlated with a CT scan, which will confirm the diagnosis. Early diagnosis and treatment were found to be associated with a lower risk of complications and mesh use during the surgical repair is an efficient mean of reducing recurrences after the surgical repair. However, the timing of repair and surgical solution still depends on the patient's condition.
\end{abstract}

Key words: Traumatic abdominal wall hernia; Traumatic abdominal wall defect; Blunt abdominal injury; Blunt abdominal trauma; Intra-abdominal injuries.
(1) Carol Davila University of Medicine and Pharmacy, Bucharest, Romania. (2) University Emergency Hospital of Bucharest, Bucharest, Romania.

Correspondence:

ANDREI MINCU

E: andreimincu.s3@gmail.com

ARTICLE INFO

Received: 14 September 2020 Accepted: 7 October 2020

\section{Introduction}

Traumatic abdominal wall hernias (TAWHs) are rare clinical conditions where muscular and fascial layers of the abdominal wall are disrupted while the skin remains intact after a blunt abdominal injury. ${ }^{1,2}$

Only a handful of cases have been reported, therefore a consensus in management of TAWHs has not been established. ${ }^{3}$

The purpose of this study was to investigate current literature regarding management of TAWHs, taking into consideration mainly circumstances of the occurrence and optimal timing for repair of hernias subsequent to blunt abdominal trauma.

\section{Method}

A thorough search through PubMed, using the following search formula: ((management OR guidelines) AND (traumatic abdominal wall hernia OR traumatic abdominal wall defect)) OR (TAWD) OR (TAWH)) was carried out. Only full text articles published in the last 13 years were chosen. After a rigorous examination, a total of 12 articles were chosen for this research.

\section{Results and Discussion}

Traumatic abdominal wall hernias are considered to be uncommon occurrences following blunt ab- 
dominal injury. The incidence of TAWHs is rather low in contrast to the more frequent blunt abdominal trauma. ${ }^{1,3-5}$ The incidence of TAWHs is estimated at $0.07 \% .^{3}$

Usually, a TAWH contains small bowel or large bowel, however a small percentage of TAWHs can contain both. Associated intra-abdominal injuries such as bowel perforation, splenic rupture, liver lacerations or pelvic fractures are frequent, the percentages varying from $30 \%$ to $60 \%{ }^{3,4,6}$ There is a possibility for the patient to become symptomatic after a longer period following the blunt abdominal trauma while only presenting mild discomfort at the site of injury. ${ }^{2}$ The location of the hernia might be linked to a higher or lower risk of concurrent visceral injuries. Supraumbilical and flank hernias pose a greater chance of being associated with intra-abdominal injuries, while infraumbilical hernias are less expected to coexist with such injuries. TAWH usually occur at weak points of the abdomen such as the lower abdomen and inguinal area and the lateral region to the rectus seath. ${ }^{4}$

Currently there is no precise classification system for TAWHs. ${ }^{4}$ Management is generally based on the mechanism and force of the injury, nature and severity of other lesions, size of the hernia and the general condition of the patient. ${ }^{6}$ TAWHs can be classified into three main types. The first type occurs in high-energy injuries, the fascial defect is large and concurrent intra-abdominal lesions are common. The second type occurs in low-energy injuries and coexisting intra-abdominal injuries are rare. The third type occurs with deceleration injuries and represents an intraabdominal herniation of the bowel., ${ }^{4}$ Ganchi et al classified these hernias based on the mechanism of injury into two major types. The "focal" type includes small hernias in which other associated injuries are uncommon. The "diffuse" type includes hernias resulted from shearing injuries and other associated injuries are more frequent. ${ }^{2,4}$

TAWHs after road accidents are commonplace, especially in motorcyclists. Such scenarios often result in "handlebar hernias", first described by Dymian in $1980 .^{4,6}$ Unsurprisingly, a high number of handlebar hernias have also been reported in children as a result of a thinner abdominal wall and more elastic skin. ${ }^{1}$ Risk factors known for TAWH are pre-existing hernias, old age and weak abdominal muscles. ${ }^{3,8}$
TAWHs can be described as a "visible bulge" on the parietal wall associated with ecchymosis and subcutaneous fluctuant swelling. 2, 3, 6 These clinical findings must be paired with computed tomography (CT) for a prompt diagnosis. ${ }^{6} \mathrm{CT}$ is considered the most accurate diagnostic tool and is able to distinguish an isolated TAWH from associated intra-abdominal injuries. ${ }^{1,4}{ }^{4}$ Even though CT is the most widely used modality of diagnosis in trauma patients, in cases where injuries call for immediate response, exploratory laparotomy will be used to evaluate the abdominal wall. ${ }^{8}$

Generally it has been accepted that prompt exploration and repair is a much more appropriate method in the management of TAWHs. ${ }^{4}$ Delayed timing of exploration and repair might be associated with bowel strangulation and excessive tension in the suturing of the defect which in itself is associated with higher recurrence rates. ${ }^{2,5,9}$ However, the timing of repair ultimately comes down to the size of the defect, timing of diagnosis and association of other intra- or extra-abdominal injuries. ${ }^{10}$ Patients who possess larger parietal defects have a lower chance of bowel strangulation thus allowing for a delayed repair of the defect. This approach will give the surrounding tissue time to heal and therefore mesh can be safely used. However, this strategy might lead to a more difficult repair due to muscle retraction and atrophy. ${ }^{10,11}$

Mesh use in the repair of TAWHs has been found to lead to less recurrences compared to no mesh repair. A recent study indicates that up to $70 \%$ of recurrences occur in patients who were treated without the use of mesh. ${ }^{12}$ If the size of the defect is small and the patient is stable, then a tension-free repair without the use of mesh during the initial exploratory laparotomy might be a feasible approach and will prevent visceral incarceration. ${ }^{8,10}$

In the presence of hollow viscus injury (HVI) or abdominal contamination, mesh use could be an option, however a study has shown that longterm durability is less favourable. ${ }^{8}$ In the past, HVIs and contaminated fields were considered absolute contraindications for the use of biologic meshes, however the issue resides more in the long-term viability of the repair rather than the safety in using of the biologic mesh in such instances. $^{6}$ 
In a recent systematic review, it has been found that tension-free repair is recommended to minimise recurrence rates. However, this might be difficult to achieve in a primary repair due to swelling and haematoma in the acute posttraumatic period. In such cases mesh use is recommended. ${ }^{12}$

\section{Conclusion}

TAWHs are rare occurrences and should be taken into consideration whenever a patient reports blunt abdominal trauma when performing the initial assessment. These hernias can often be associated with intra-abdominal injuries and computed tomography should be a standard diagnostic tool. Early repair is associated with a lower risk of complications and mesh use has been proven to be an efficient method of minimising recurrences after the surgical repair. In cases where the size of the defect is large, the repair procedure must involve the use of mesh. If the size of the defect is small, it can be handled with a tension-free repair without the use of mesh.

\section{References}

1. Pathak D, Mukherjee R, Das P, Pathak D, Gangopadhyay A, Das S. Traumatic abdominal wall hernia with concealed colonic perforation. Ann R Coll Surg Engl 2016;98(7):e133-5. doi: 10.1308/rcsann.2016.0178.

2. Singal R, Gupta R, Mittal A, Gupta A, Singal RP, Singh B, et al. Delayed presentation of the traumatic abdominal wall hernia; dilemma in the management - review of literature. Indian J Surg 2012 Apr;74(2):149-56.

3. Suhardja TS, Atalla MA, Rozen WM. Complete abdominal wall disruption with herniation following blunt injury: case report and review of the literature. Int Surg 2015 Mar;100(3):531-9.

4. Choi HJ, Park KJ, Lee HY, Kim LH, Kim SH, Kim MC, et al. Traumatic Abdominal Wall Hernia (TAWH): a case study highlighting surgical management. Yonsei Med J 2007 Jun;48(3):549-53.

5. Constantin V, Carâp A, Bobic S, Albu M, Nica E, Socea B. Traumatic abdominal wall hernia associated with small bowel injury-case report. Indian J Surg 2015 Apr 1;77(Suppl 1):174-6.

6. Ballard DH, Kaskas NM, Hamidian Jahromi A, Skweres J, Youssef AM. Abdominal wall hernia and aortic injury secondary to blunt trauma: case report and review of the literature. Int J Surg Case Rep 2014;5(12):1238-41.

7. Aravinda PS, Sudipta S, Saurabh G, Andley M, Kumar A. Traumatic Spigelian hernia: a rare clinical scenario. J Clin Diagnostic Res 2014;8(5):4-5.

8. Jahromi AH, Skweres J, Sangster G, Johnson L, Samra N. What we know about management of traumatic abdominal wall hernia: review of the literature and case report. Int Surg 2015 Feb;100(2):233-9.

9. Yücel N, Uğraş MY, Ișık B, Turtay G. Case report of a traumatic abdominal wall hernia resulting from falling onto a flat surface. Ulus Travma Acil Cerrahi Derg [Internet]. 2010 Nov [cited 2020 Apr 30];16(6):5714. Available from: http://www.ncbi.nlm.nih.gov/ pubmed/21153955.

10. Al Beteddini OS, Abdulla S, Omari O. Traumatic abdominal wall hernia: a case report and literature review. Int J Surg Case Rep 2016;24:57-9. doi: 10.1016/j. ijscr.2016.03.038.

11. Yadav S, Jain SK, Arora JK, Sharma P, Sharma A, Bhagwan J, et al. Traumatic abdominal wall hernia: delayed repair: advantageous or taxing. Int J Surg Case Rep 2013;4(1):36-9. doi: 10.1016/j.ijscr.2012.10.004.

12. Karhof S, Boot R, Simmermacher RKJ, Van Wessem KJP, Leenen LPH, Hietbrink F. Timing of repair and mesh use in traumatic abdominal wall defects: a systematic review and meta-analysis of current literature. World J Emerg Surg 2019 Dec 17;14:59. doi: 10.1186/s13017-0190271-0.

In regard to the timing of TAWH repair, early repair seems to be associated with a decreased risk
of bowel obstruction, strangulation or incarceration. If all of the aforementioned risks are absent and the patient's status is acceptable, a delayed

\section{Acknowledgements}

None.

\section{Conflict of interest}

None. 